\title{
Magnetization of Steel Building Materials and Structures in the Natural Geomagnetic Field
}

E. Čermáková

This paper presents the physical basis of the magnetic properties of ferromagnetic materials and shows their relationships with external geomagnetic field. It graphically processes the experimental data detected by an HMR magnetometer. Taking into account the natural geomagnetic field under the effects of steel $U$ profiles, variations of the natural geomagnetic field in a steel structure building are indicated and the potential existence of Sick Building Syndrome (SBS) in these types of buildings is pointed out.

Keywords: experiment, geomagnetic fields, magnetometer, Sick Building Syndrome, U profile.

\section{Introduction}

Of fundamental significance from the microscopic point of view, for the magnetisation of metals (including steel) is the relationship that couples the total moment of momentum $J$ and the total magnetic moment $\boldsymbol{m}_{t o t}$, the electronic envelope of the atom. The total magnetic moment is here not an integral of movement and, considered from the point of view of quantum mechanics, is not a factor simultaneously measurable with the power and the total moment of the atom momentum. What is called the effective magnetic moment $\boldsymbol{m}_{G}$ is therefore established as a projection of the vector $\boldsymbol{m}_{t o t}$ into the direction of the opposite vector $J$, which is sufficient to describe the basic magnetic phenomena given by the relationship between vectors $\boldsymbol{m}_{G}$ and $\boldsymbol{J}$.

Vector $\boldsymbol{J}$ (and also $\boldsymbol{m}_{G}$ ) can take only discrete positions in the direction of the magnetic field (the projections $J_{H}$ of vector $\boldsymbol{J}$ in the direction of field $\boldsymbol{H}$ are given as multiples of Planck's constant $\hbar=h /(2 \pi)$, in the form $J_{H}=M_{G} \hbar ; M_{G}$ is the quantum number for the projection of the total moment of the atom momentum into the axis of quantization. Projections $m_{G H}$ of vector $\boldsymbol{m}_{G}$ in the direction of field $\boldsymbol{H}$ are also possible. Then the energy $E_{H}$ of the magnetic moment $\boldsymbol{m}_{G}$ in the external magnetic field $H$ is of the form

$$
E_{H}=\mu_{0} m_{G H}|\boldsymbol{H}|
$$

and indicates the potential levels of energy of the atom in the magnetic field.

The level of atom energy, given by a single definite value in the initial spherically symmetric field of an atom, splits up in the magnetic field. The creation of a magnetic moment which fixes the magnetic behaviour of the substances is accomplished only by non-closed electron shells. The non-closed shell of ferromagnetic materials is exposed to strong effects of internal fields in the crystal lattice. This situation must be reckoned with in accounting for the characteristics of elements in the iron group.

When accounting for the magnetic characteristics of solid substances, especially metals, it is also necessary to consider, as a consequence of spin-orbital coupling, the orbital/magnetic moment to different extents depending on the way the electron is coupled by its orbital movement to the crystal lattice. The result of this phenomenon is, on the one hand, magnetic crystal anisotropy, i.e. various positions of the magnetic mo- ments to the crystal lattice which are not energetically equivalent, and, on the other hand, magnetostriction, i.e. there appears a spontaneous deformation of the crystal lattice with varying magnetic states.

Macroscopically, the external magnetic field influencing some ferromagnetic material is, in our case, the geomagnetic field. This field can be described by the vector of intensity of the geomagnetic field $\boldsymbol{H}$ for any point in space as a gradient of geomagnetic potential $W$.

$$
\boldsymbol{H}=-\operatorname{grad} W,
$$

where $W$ is a function of the coordinates meeting Laplace's equation $\Delta W=0$.

The vector of geomagnetic induction $\boldsymbol{B}$ is physically coupled with the vector of intensity of the geomagnetic field $\boldsymbol{H}$ by the relationship

$$
\boldsymbol{B}=\mu \boldsymbol{H}
$$

where $\mu$ is permeability of the environment.

When considering a vector of the magnetic field experimentally detected at any point in space, it is necessary to consider the resultant vector $\boldsymbol{H}$ with mutually perpendicular components $H_{x}, H_{y}, H_{z}$, where the resultant values of the vector is given by the formula

$$
|\boldsymbol{H}|=\sqrt{H_{x}^{2}+H_{y}^{2}+H_{z}^{2}}
$$

or, for the vector of magnetic induction

$$
|\boldsymbol{B}|=\sqrt{B_{x}^{2}+B_{y}^{2}+B_{z}^{2}} .
$$

The geomagnetic field described above magnetizes ferromagnetic materials (involving various alloy steels, cast iron, steel reinforcement of panels, various types of building steels) which become magnetized even by weak magnetic fields and keep this condition also after the external magnetic field has been removed.

\section{Experiment}

\subsection{Measuring instruments}

Measurement of minor deformations of the geomagnetic field requires sensitive magnetometers. We used a three-axis smart digital magnetometer (HMR) which detects the strength and direction of a magnetic field and communicates 
the $\mathrm{x}, \mathrm{y}$, and $\mathrm{z}$ component directly to a computer. Three independent bridges are oriented to sense the $\mathrm{x}, \mathrm{y}$, and $\mathrm{z}$ axis of a magnetic field. The bridge outputs are then converted to a 16-bit digital value using an internal delta-sigma $\mathrm{A} / \mathrm{D}$ converter. A command set configures the data sample rate, output format, averaging and zero offset. An on-board EEPROM stores any configuration changes for next time power-up. Other commands perform utility functions like band rate, device ID and serial number. Also included in the HMR magnetometer is a digital filter with $50 / 60 \mathrm{~Hz}$ rejection to reduce the ambient magnetic interference.

A unique switching technique is applied to the permalloy bridge to eliminate the effects of past magnetic history. This technique cancels out the bridge offset as well as any offset introduced by the electronics. The $\mathrm{x}, \mathrm{y}$, and $\mathrm{z}$ digitized data is sent out as a series of bytes, either after an ID match is received from the control processor, or as a continuous data stream. The data is serially output in either the 9,600 band or the 19,200 band, using the RS-232 or RS-485 standard, for serial input to most personal computers. The RS-485 standard allows up to 32 devices to be connected on a single wire pair up to 4,000 feet in length. An HMR address can be stored in the on-board EEPROM to assign one of thirty-two unique ID codes to allow direct line access. An internal microcontroller handles the magnetic sensing, digital filtering, and all output communications, eliminating the need for external trims and adjustments. Standard RS-485 or RS-232 drivers provide compliant electrical signalling.

HMR magnetometers provide

- A range of $\pm=2 \cdot 10^{-4} \mathrm{~T}:<7 \cdot 10^{-9} \mathrm{~T}$ Resolution

- High Accuracy over $\pm 1 \cdot 10^{-4} \mathrm{~T}:<0.5 \%$

- Output Rate Selectable: 10 to 154 Samples/Sec.

- Three-Axis Digital Output: BCD ASCII or Binary

- RS-232 or RS-485 Serial Output.

\subsection{Results of experiments}

The outcome of detection of the natural geomagnetic field in the open air is a vector of magnetic induction $\boldsymbol{B}$ (see Eq. 3 and 5), which moves in our geographical latitudes around $50 \mu \mathrm{T}$ under the effects of daily and long-time variations and non-homogeneous distribution of ferromagnetic materials in the Earth crust. This value oscillates slightly in $\mu \mathrm{T}$ units. The magnetic induction measured in wood buildings (and classic brick buildings) without the presence of ferromagnetic materials (building steels) was almost the same as in the open air. Fig. 1 shows the magnetic induction on the floor of a classic brick building, in an unfurnished room, at a sufficient distance from the piping which, in older buildings, is mostly cast iron (building steel). (In modern buildings the pipes are made of plastic materials or light metals that do not deform the natural geomagnetic fields.)

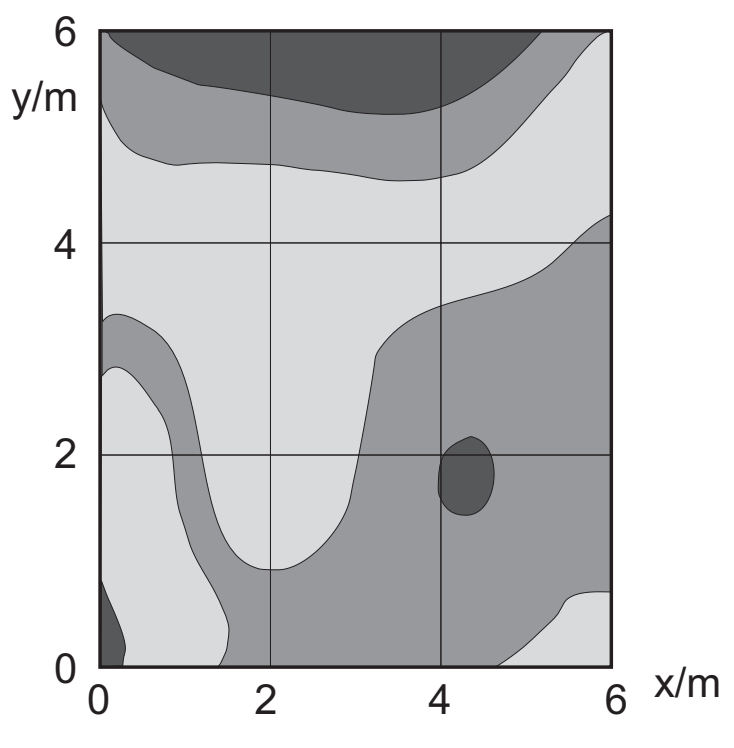

\section{$\mathrm{B} / 10^{-5} \mathrm{~T}$}

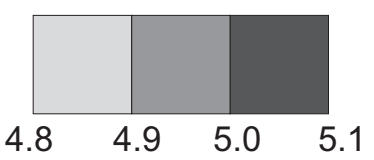

Fig. 1: Magnetic induction on the floor of a classic brick building

Classic brick buildings of older type all had a reinforcement, including the roof trusses, made of wood materials that do not influence the form of the geomagnetic field. At the present time, the building industry favours so-called clay buildings constructed with the use of modern technologies. The geomagnetic field in these buildings is similar to that of Fig. 1.

However, when we use any kind of building steel in the construction - e.g. typical U profiles (ceilings and floors of rooms, see Fig. 2), these materials become magnetized and deform the natural geomagnetic field.

Ferromagnetic materials inserted into a magnetic field modify the form and concentration of the magnetic lines of force which are retransformed into the initial condition at a certain distance from the ferromagnetic material. The above-mentioned magnetometer was used to measure the deformation of the natural geomagnetic field in the direction of the geomagnetic meridian (see Fig. 3) and in a direction deviating by $35^{\circ}$ from the geomagnetic meridian. To find the
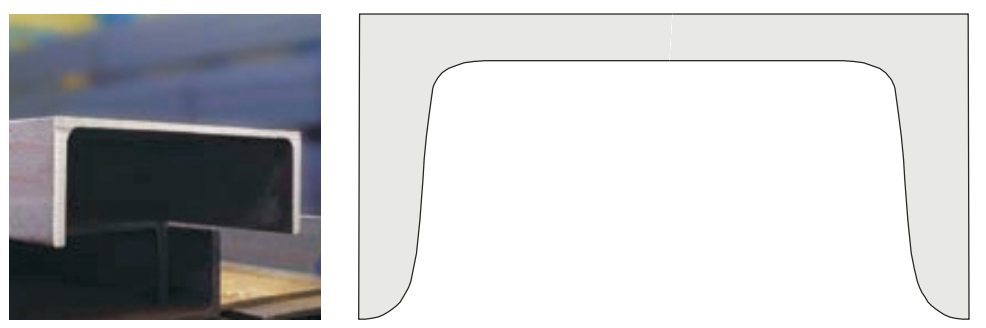

Fig. 2: U profile currently used in the building industry 


\section{$\mathrm{U}$ profile $0^{\circ}$}

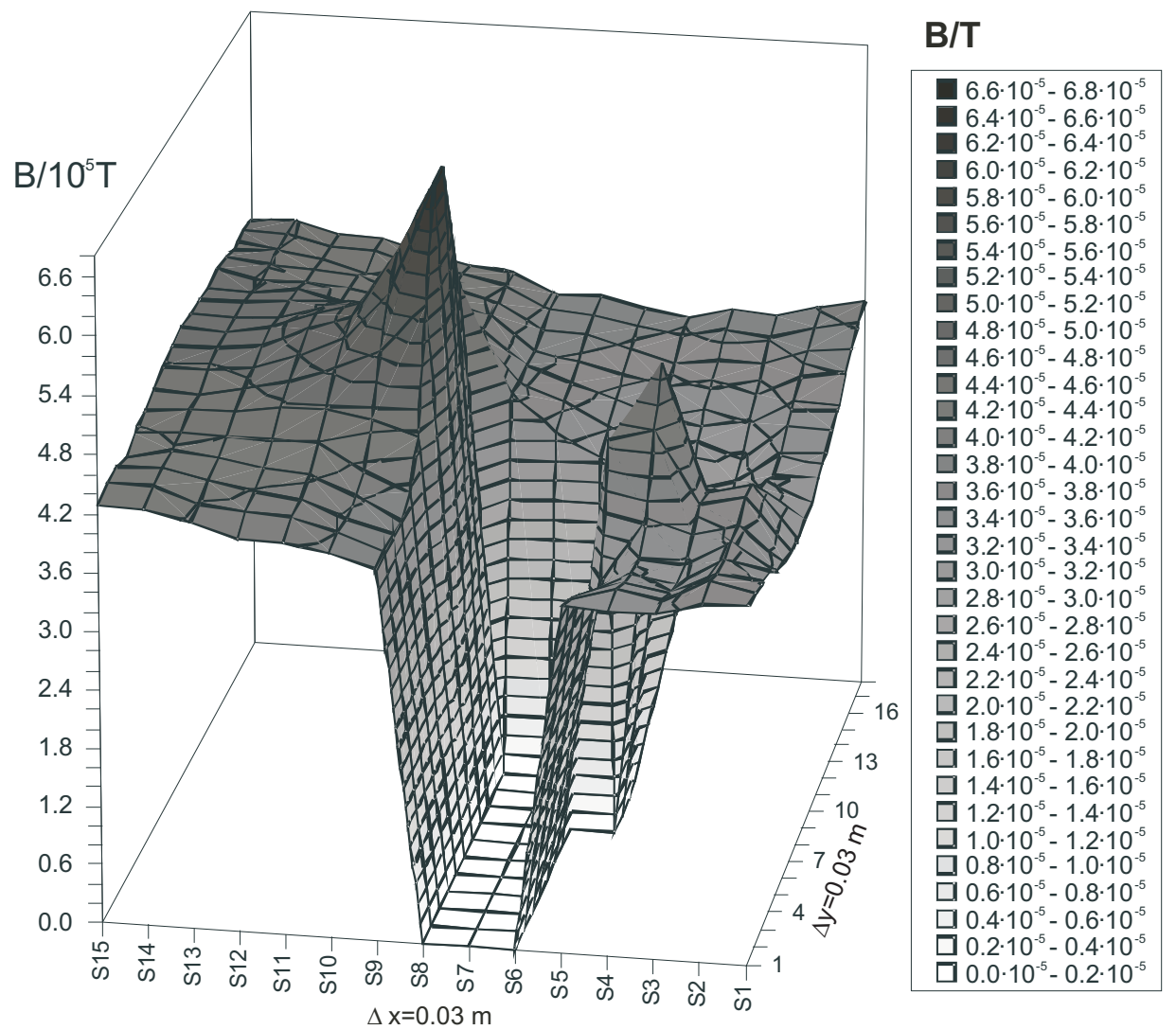

Fig. 3: Deformation of the geomagnetic field due to a $U$ profile laid in the direction of the geomagnetic meridian

\section{$\mathrm{U}$ profile $35^{\circ}$}

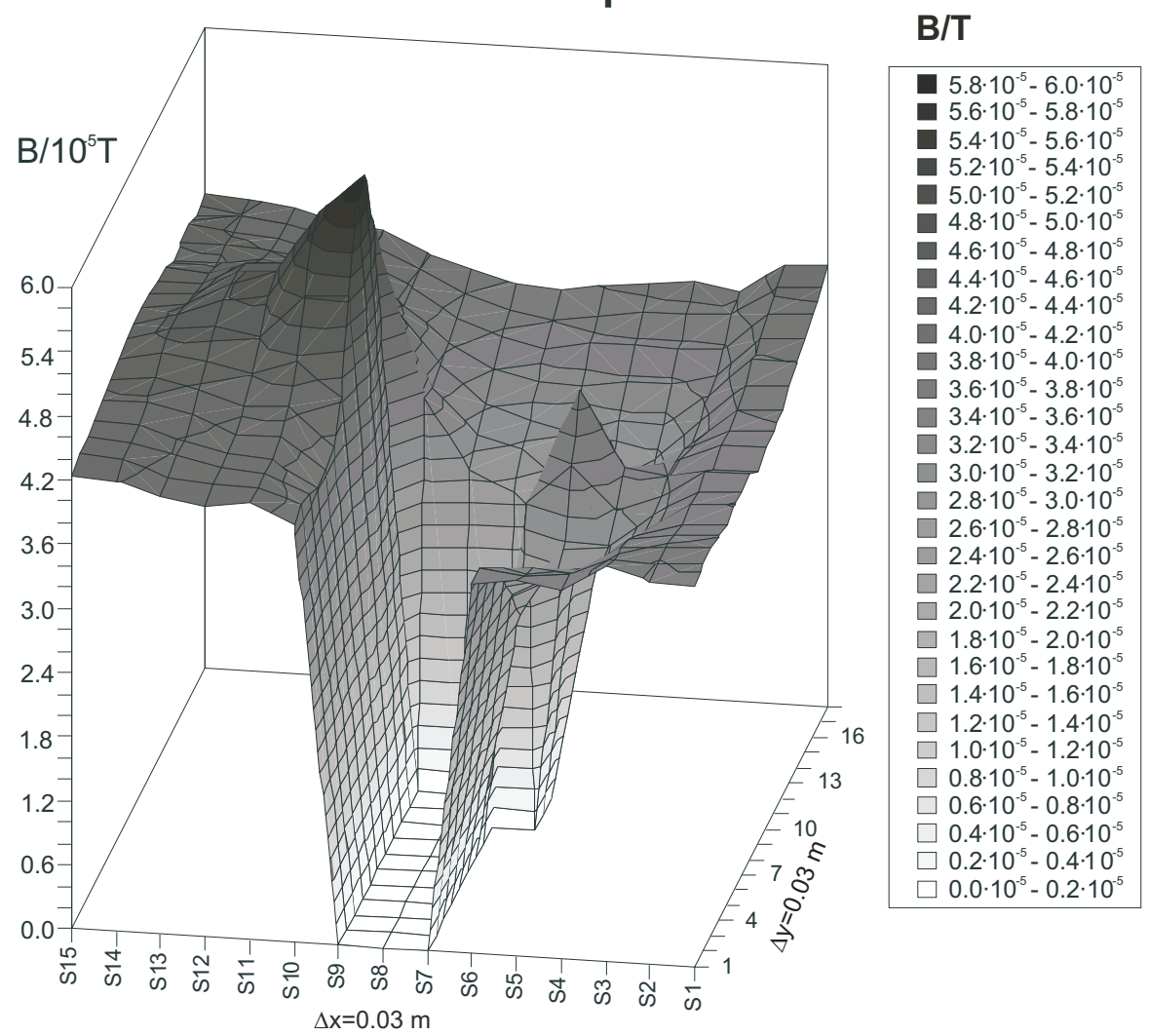

Fig. 4: Deformation of the geomagnetic field due to a U profile laid in a direction deviated by $35^{\circ}$ away from the direction of the geomagnetic meridian 


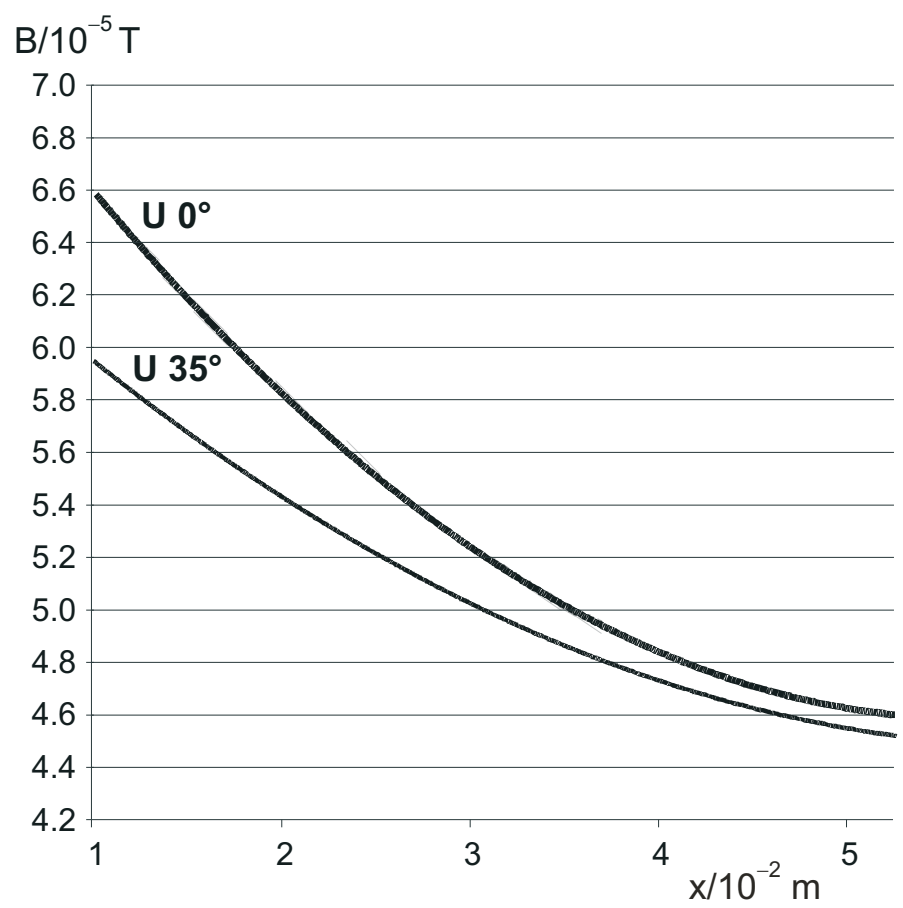

Fig. 5: Change in magnetic induction as a function of the distance of the ferromagnetic material

declination, i.e. the congruent and deviated inclination to the geomagnetic meridian, a geodetic compass was used.

Fig. 3 shows maximum deformation of the geomagnetic field within the interval $(0.2-6.8) \cdot 10^{-5} \mathrm{~T}$ with the biggest deformation taking place along the edges of the $\mathrm{U}$ profile and in the place of the maximum cluster of steel. Fig. 4, too, clearly shows maximum deformation of the natural geomagnetic field within the interval $(0.2-6.0) \cdot 10^{-5} \mathrm{~T}$, with the biggest deformation taking place along the edges of the U profile and in the place of the maximum cluster of steel. The total change in magnetic induction is $0.8 \cdot 10^{-5} \mathrm{~T}$ less than that for the congruent inclination to the geomagnetic meridian, and is given by the declination away from the direction of the main geomagnetic meridian. This deformation and change in the magnetic field decreases appreciably with distance from the ferromagnetic body that causes this deformation. See Fig. 5 .

The steel skeleton of the building also contributes considerably to the deformation of the natural magnetic field. Fig. 6 represents the steel skeleton of a part of a building which limits off a hall. The areas inside the steel skeleton are filled with reinforced panels. The measurement and graphical processing of the magnetic induction in a building with a steel skeleton is represented in Fig. 7.

The measurement of the hall in the steel structure was time-consuming and laborious. The HMR magnetometer was used to detect magnetic induction in steps $(0.5 \times 0.5) \mathrm{m}$ over areas parallel and $0.5 \mathrm{~m}$ apart from each other. To give a better idea of the changes in the magnetic field (magnetic in-

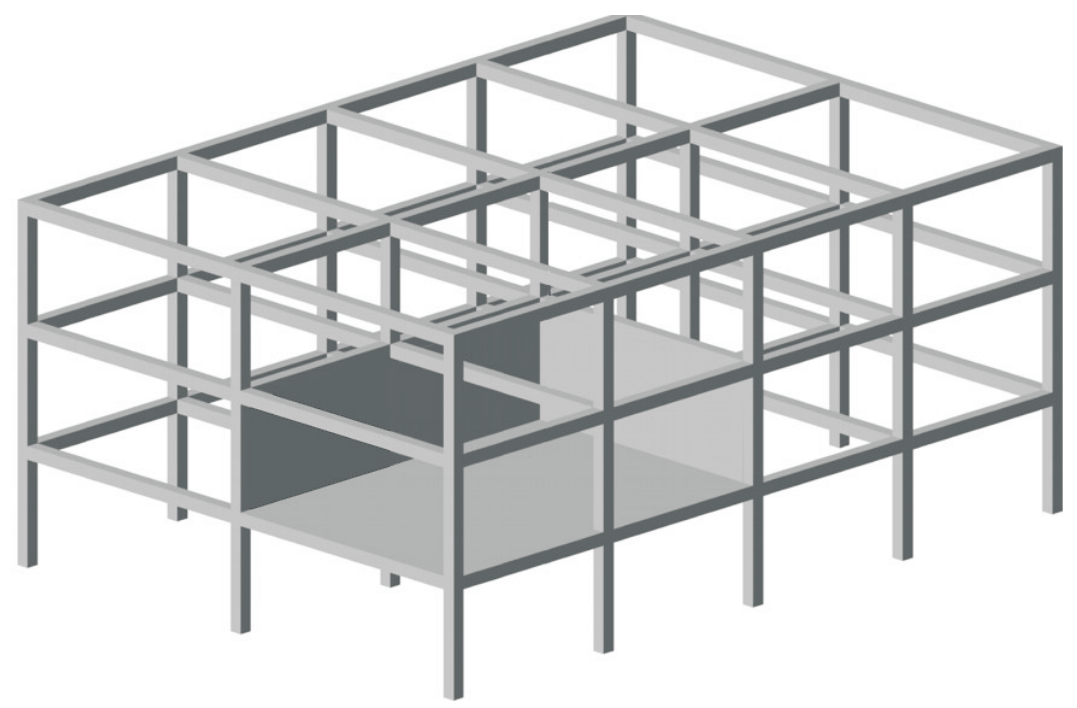

Fig. 6: Steel skeleton of a part of a building filled with reinforced panels 


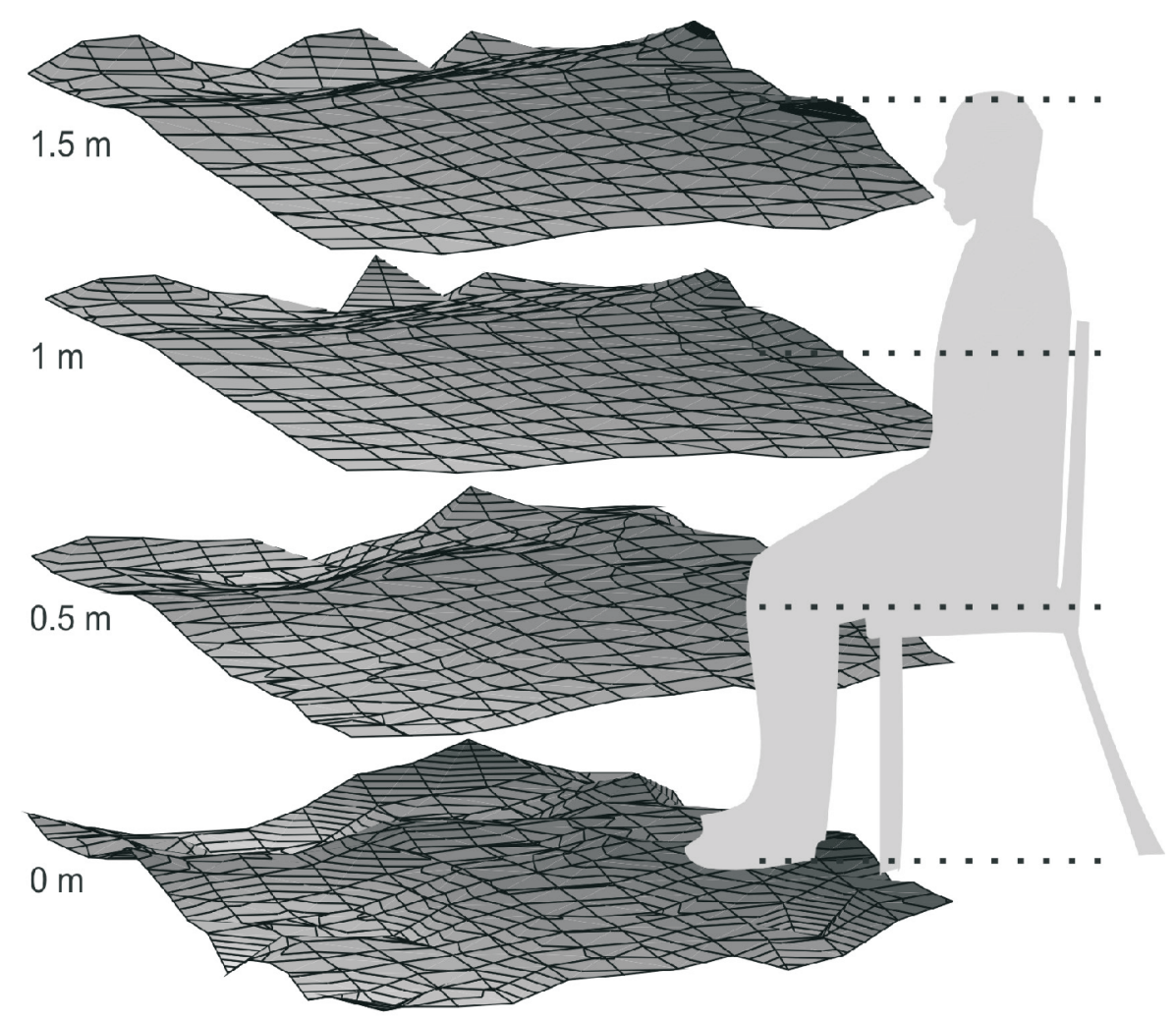

$\begin{array}{llllllllllllll}1.8 & 2.2 & 2.6 & 3.0 & 3.4 & 3.8 & 4.2 & 4.6 & 5.0 & 5.4 & 5.8 & 6.2 & 6.6 & \mathrm{~B} / 10^{-5} \mathrm{~T}\end{array}$

Fig. 7: Deformation of the geomagnetic field in a building with a steel skeleton and panel fillings

duction) the values measured include the figure of a sitting man. To allow comparisons, the variation in magnetic induction for $1 \mathrm{~m}$ height is added (Fig. 8).

\section{Conclusion}

The paper shows appreciable changes in the natural geomagnetic fields (magnetic induction) due to building steel (ferromagnetic material), namely reinforcing $\mathrm{U}$ profiles, and overall effects of steel structures on the geomagnetic field inside a building (a hall on the second floor of a building). These changes and deformations of the geomagnetic field can contribute substantially to Sick Building Syndrome. Long-time development has adapted man to the geomagnetic field. The houses he inhabited in the past were constructed with classic materials such as wood, clay and burnt bricks, which do not deform the natural geomagnetic fields. However, the present time has brought mostly high-rise buildings of a technical character, which greatly change the magnetic field that man has been adapted to. When working in such an environment, some people develop non-specific types of diseases such as increased fatigue, reduced immunity, poor concentration and the like. The building under test gives rise to changes of magnetic induction as high as $30 \mu \mathrm{T}$. This is a value that is not taken into account or regu- lated by any hygienic standard. As people nowadays spend much shorter times in the open air and live as much as $90 \%$ of the day in enclosed spaces, it is a question of time before even these relatively small changes in stationary magnetic fields will be regulated.

\section{Acknowledgment}

The author thanks the Ministry of Education of the Czech Republic for its financial support for this work within the framework of project CEZ: J22/98:261100007.

\section{References}

[1] Kittel, Ch.: Introduction to Solid State Physics. $6^{\text {st }}$ ed. New York: J. Willey 1981, 218p.

[2] Moon, F.: Magneto-Solid Mechanics. $2^{\text {nd }}$ ed. New York: Cornell University, 1995, 435 p., ISBN 0-471-88536-3.

[3] Mareš, S.: Introduction to the Applied Geophysics (in Czech), $2^{\text {nd }}$ ed. Praha: SNTL 1990, 677 p., ISBN 80-03-00427-6.

[4] Božáková, M., Čermáková, E., Černý, J.: “Deformation of Geomagnetic Field in a Building with a Steel Reinforcement." In: Proceedings of Czech and Slovak Conference Experiment 04, 14-15. X, 2004 Brno. Brno: ed. CERM, s.r.o. 2004, p. 55-57. ISBN 80-7204-354-4. 


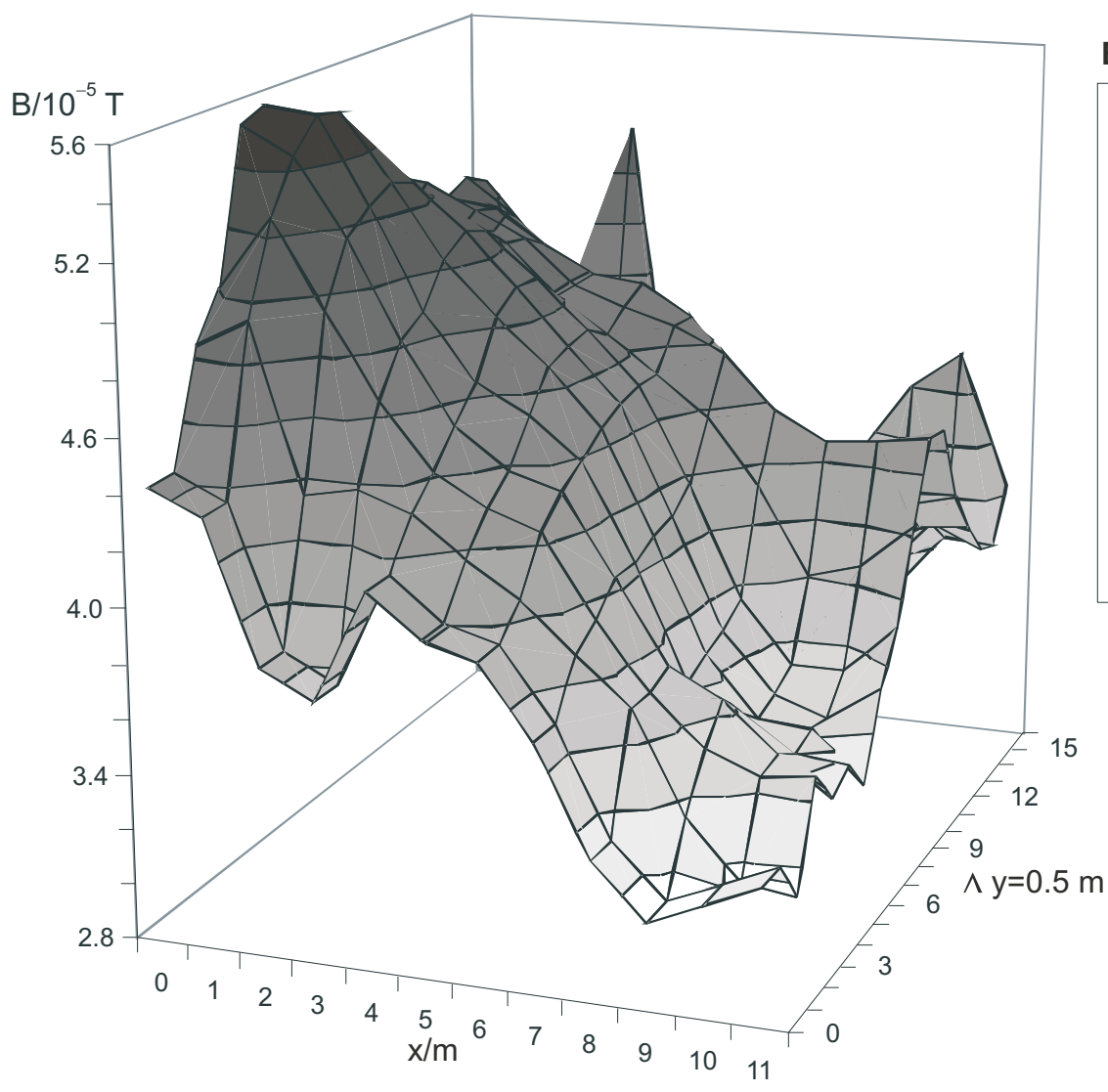

$\mathrm{B} / \mathrm{T}$

口 $5.4 \cdot 10^{-5}-5.6 \cdot 10^{-5}$

- $5.2 \cdot 10^{-5}-5.4 \cdot 10^{-5}$

口 $5.0 \cdot 10^{-5}-5.2 \cdot 10^{-5}$

प $4.8 \cdot 10^{-5}-5.0 \cdot 10^{-5}$

$\square 4.6 \cdot 10^{-5}-4.8 \cdot 10^{-5}$

प $4.4 \cdot 10^{-5}-4.6 \cdot 10^{-5}$

ㅁ $4.2 \cdot 10^{-5}-4.4 \cdot 10^{-5}$

ㅁ $4.0 \cdot 10^{-5}-4.2 \cdot 10^{-5}$

ㅁ $3.8 \cdot 10^{-5}-4.0 \cdot 10^{-5}$

ㅁ $3.6 \cdot 10^{-5}-3.8 \cdot 10^{-5}$

ㅁ $3.4 \cdot 10^{-5}-3.6 \cdot 10^{-5}$

$\square 3.2 \cdot 10^{-5}-3.4 \cdot 10^{-5}$

$\square 3.0 \cdot 10^{-5}-3.2 \cdot 10^{-5}$

$\square 2.8 \cdot 10^{-5}-3.0 \cdot 10^{-5}$

Fig. 8: Changes in magnetic induction for $1 \mathrm{~m}$ heights in the hall

[5] Čermáková, E., Žampach, R.: "Geomagnetic Field Deformation in the Steel-Structured Buildings." In: Proceedings of $10^{\text {th }}$ International Scientific Conference CO-MAT-TECH 2002 - Applied Natural and Engineering Science. Bratislava: ed. STU Bratislava 2002, p. 260-263, ISBN 80-227-1768-1.

[6] Collective: Dictionary of Sun - Earth Atmosphere. (in Slovak) Bratislava: Slovak publisher AV Bratislava 1991, 274 p.
RNDr. Eleonora Čermáková, CSc. phone: +420541 147662 fax: +420541 147666 e-mail: cermakova.e@fce.vutbr.cz Department of Physics

Brno University of Technology Faculty of Civil Engineering Žižkova 17

60200 Brno, Czech Republic 